Supplement of The Cryosphere, 10, 2533-2539, 2016

http://www.the-cryosphere.net/10/2533/2016/

doi:10.5194/tc-10-2533-2016-supplement

(C) Author(s) 2016. CC Attribution 3.0 License.

(c) (1)

Supplement of

\title{
Brief communication: Organochlorine pesticides in an archived firn core from Law Dome, East Antarctica
}

Marie Bigot et al.

Correspondence to: Marie Bigot (mbigot1@ gmail.com)

The copyright of individual parts of the supplement might differ from the CC-BY 3.0 licence. 
Table 1: Surrogate standard recoveries for ice core sample A and B (in \%)

\begin{tabular}{|c|c|c|c|c|}
\hline \multirow{2}{*}{$\begin{array}{r}\text { Sampling material } \\
\text { Sample \# }\end{array}$} & \multicolumn{2}{|c|}{$\begin{array}{c}\text { XAD } \\
\text { (dissolved } \\
\text { compounds) } \\
\end{array}$} & \multicolumn{2}{|c|}{$\begin{array}{c}\text { Glass fibre filter } \\
\text { (particles) }\end{array}$} \\
\hline & $\mathbf{A}$ & B & $\mathbf{A}$ & B \\
\hline Hexachlorobenzene, ${ }^{13} \mathrm{C}_{6^{-}}$ & 15 & 18 & 17 & 6 \\
\hline$\alpha-\mathrm{HCH},{ }^{13} \mathrm{C}_{6^{-}}$ & 46 & 53 & 47 & 50 \\
\hline$\gamma$-HCH-D6 & 30 & 39 & 48 & 51 \\
\hline Heptachlor, ${ }^{13} \mathrm{C}_{10^{-}}$ & 130 & 128 & 77 & 80 \\
\hline Oxychlordane, ${ }^{13} \mathrm{C}_{10^{-}}$ & 49 & 56 & 64 & 64 \\
\hline trans-Nonachlor, ${ }^{13} \mathrm{C}_{10^{-}}$ & 63 & 66 & 51 & 59 \\
\hline Dieldrin, ${ }^{13} \mathrm{C}_{12^{-}}$ & 47 & 55 & 52 & 58 \\
\hline Endrin, ${ }^{13} \mathrm{C}_{12-}$ & 49 & 67 & 72 & 82 \\
\hline Endosulfan II, ${ }^{13} \mathrm{C}_{9}$ - & 22 & 34 & 43 & 49 \\
\hline $4,4^{\prime}-$ DDE, ${ }^{13} \mathrm{C}_{12^{-}}$ & 36 & 42 & 43 & 46 \\
\hline 4,4'-DDD, ${ }^{13} \mathrm{C}_{12^{-}}$ & 14 & 21 & 42 & 42 \\
\hline $4,4^{\prime}$-DDT, ${ }^{13} \mathrm{C}_{12^{-}}$ & 8 & 11 & 40 & 36 \\
\hline Methoxychlor-D6 & 102 & 168 & 37 & 36 \\
\hline Mirex, ${ }^{13} \mathrm{C}_{10^{-}}$ & 5 & 5 & 21 & 18 \\
\hline Mirex, ${ }^{13} \mathrm{C}_{8^{-*}}{ }^{*}$ & 7 & 6 & 30 & 28 \\
\hline$\delta$-HCH* & 135 & 67 & 96 & 110 \\
\hline
\end{tabular}

Notes: * indicates surrogate standards added prior to sampling (XAD) or prior to extraction (filters)

Table 2 Concentrations and deposition rates of detected OCPs in Law Dome firn core, sample A (1945-57) and sample B (1958-67)

\begin{tabular}{rccccc}
\cline { 2 - 6 } & \multicolumn{2}{c}{ Sample A } & \multicolumn{2}{c}{ Sample B } \\
\cline { 2 - 6 } & MDL & Concentration & $\begin{array}{c}\text { Deposition } \\
\text { rate }\end{array}$ & Concentration & $\begin{array}{c}\text { Deposition } \\
\text { rate }\end{array}$ \\
\hline $\boldsymbol{\alpha}$-HCH & 0.87 & 22 & 0.24 & 19 & $\mathrm{pg} \cdot \mathrm{cm}^{-2} \mathrm{yr}^{-1}$ \\
$\boldsymbol{\gamma}$-HCH & 1.18 & 22 & 0.24 & 60 & 0.25 \\
Heptachlor & 0.51 & 2.3 & 0.03 & 2.4 & 0.78 \\
trans-Chlordane & 1.25 & $<3.3$ & - & 5.0 & 0.03 \\
Dieldrin & 4.75 & 222 & 2.4 & 310 & 0.07 \\
Endrin & 1.20 & 4.8 & 0.05 & 8.3 & 4.0 \\
\hline
\end{tabular}

Notes: MDL stands for method detection limits, < indicates values detected below limits of quantifications (determined for each individual sample). 